\title{
Fuzzy Quantification in Two Real Scenarios: Information Retrieval and Mobile Robotics
}

\author{
Félix Díaz-Hermida, ${ }^{*}$ Alberto Bugarín ${ }^{\dagger}$ Purificación Cariñena, ${ }^{\ddagger}$ \\ Manuel Mucientes, ${ }^{\S}$ David E. Losada" \\ Departamento de Electrónica e Computación, Universidade de Santiago de \\ Compostela, Santiago de Compostela, Spain
}

Fuzzy quantification supplies powerful tools for handling linguistic expressions. Nevertheless, its advantages are usually shown at the theoretical level without a proper empirical validation. In this work, we review the application of fuzzy quantification in two application domains. We provide empirical evidence on the adequacy of fuzzy quantification to support different tasks in the context of mobile robotics and information retrieval. This practical perspective aims at exemplifying the actual benefits that real application can get from fuzzy quantifiers. (c) 2009 Wiley Periodicals, Inc.

\section{INTRODUCTION}

Modeling of fuzzy quantification operators is a topic that has been widely discussed by a number of authors, ${ }^{1-12}$ presenting different models that can be used to relax the interpretation of classical existential and universal quantifiers, linguistically oriented quantifiers (e.g., "three or more," "more than eighty per cent," etc.) and quantifiers used for representing statistical indexes (e.g., overlapping indexes).

Following Zadeh, ${ }^{12}$ fuzzy linguistic quantifiers can be used to represent absolute or proportional fuzzy quantities (e.g. "about three men are tall" and "about 70\% of blonde men are tall," respectively). The usual application of these quantification models has been the evaluation of fuzzy-quantified statements (FQS), where the fulfillment of a number of fuzzy properties by the elements of the domain is expressed by means of a quantifier. Among other fields of application, FQS have been used for flexible queries handling both in data retrieval ${ }^{2}$ or information retrieval. ${ }^{13,14}$ In these domains, the ability of FQS for defining query languages that are natural and intuitive for real users is found to be very important. FQS are also very useful in data mining applications, ${ }^{15}$ where the main advantages come from the capabilities

\footnotetext{
*Author to whom all correspondence should be addressed; e-mail: felix.diaz@usc.es.

†e-mail: alberto.bugarin.diz@usc.es.

‡e-mail: puri.carinena@usc.es.

$\S$ e-mail: manuel.mucientes@usc.es.

Ile-mail: david.losada@usc.es.
}

INTERNATIONAL JOURNAL OF INTELLIGENT SYSTEMS, VOL. 24, 572-586 (2009)

(C) 2009 Wiley Periodicals, Inc. Published online in Wiley InterScience (www.interscience.wiley.com). • DOI 10.1002/int.20349 
of FQS for managing different levels of information granularity. FQS have also been applied in knowledge reasoning and control systems ${ }^{16}$ to facilitate the acquisition of expert knowledge.

In this work, we present two applications for fuzzy quantification both in two demanding domains: information retrieval and temporal fuzzy control in mobile robotics. Our aim here is to show how fuzzy quantification models are useful for other fields of use different of the usual ones.

In Section 2, a brief review of some of the most relevant fuzzy quantification models is presented. Section 3 describes how to apply fuzzy quantification in information retrieval (IR) for large collections of documents. FQS supply appropriate formal tools for handling linguistic expressions, which enrich the query language of the IR system. The key ideas of the model used are sketched, and the main experimental results are reported.

In Section 4, the implementation of two fuzzy control systems for the implementation of the obstacle avoidance and wall following behaviors in mobile robotics is described. Modeling of the knowledge-based control systems for these behaviors is made using fuzzy temporal quantified statements that allow a temporal evolution of the occurrence of events to be estimated. The paper ends with some conclusions.

\section{FUZZY QUANTIFICATION MODELS}

Most models in the literature follow Zadeh's approach to fuzzy quantification, ${ }^{12}$ which is very useful from a practical point of view. Nevertheless, it does not comprise some families of quantified sentences, such as quantified sentences of exception (e.g., "all except 3 students are tall"), or comparative sentences (e.g., "there are about 3 more tall people than blond people"). Zadeh's model has also been criticized due to the lack of fulfillment of relevant properties such as coherence with logic, continuity, or antonym. ${ }^{1,6,8}$ However, its implementation is simple and its underlying semantics is clear.

Other proposals have also been described in the literature. Yager's inclusive model ${ }^{17}$ fails to fulfill very relevant properties for propositions involving two fuzzy properties (type II propositions), such as duality or local monotonicity, while Yager's OWA model ${ }^{18}$ does not fulfill monotonicity. Other methods based on cardinality measures have been defined, ${ }^{3}$ although relevant properties such as monotonicity, correct generalization, or continuity are also missing in some particular cases.

Therefore, none of these models are capable to fulfill all of the properties that may be defined as rational for a fuzzy quantifier. Summing up, the selection of the most appropriate model for a given application must be preceded by a careful analysis of which properties are really relevant for that application. Only the models that fulfill such properties should be candidates as good quantification models for that application, thus producing a consistent modeling of FQS.

A different approach based on the concept of semifuzzy quantifier was defined $^{6-8}$ to overcome the limitations of the previous approaches. Semifuzzy quantifiers were formally defined as 
DEFINITION $1 .^{6-8}$ An n-ary semifuzzy quantifier $F Q$ on a base set $E \neq \varnothing$ is a mapping $F Q: \mathcal{P}(E)^{n} \longrightarrow[0,1]$ which to each choice of crisp $X_{1}, \ldots, X_{n} \in \mathcal{P}(E)$ assigns a gradual result $F Q\left(X_{1}, \ldots, X_{n}\right) \in[0,1]$.

Examples of semifuzzy quantifiers are ${ }^{\mathrm{a}}$

$$
\begin{aligned}
& \text { about_5 }\left(X_{1}, X_{2}\right)=T_{2,4,6,8}\left(\left|X_{1} \cap X_{2}\right|\right) \\
& \text { about } 80 \% \text { or_more_of_the }\left(X_{1}, X_{2}\right)=\left\{\begin{array}{l}
S_{0.5,0.8}\left(\frac{\left|X_{1} \cap X_{2}\right|}{\left|X_{1}\right|}\right) X_{1} \neq \varnothing \\
1
\end{array}\right. \\
& \text { about5_more_than }\left(X_{1}, X_{2}\right)=T_{1,4,6,9}\left(\left|X_{1}\right|-\left|X_{2}\right|\right) \\
& \text { nearly_equal }\left(X_{1}, X_{2}\right)= \begin{cases}S_{0.5,0.8}\left(\frac{\left|X_{1} \cap X_{2}\right|}{\left|X_{1} \cup X_{2}\right|}\right) & X_{1} \cup X_{2} \neq \varnothing \\
1 & X_{1} \cup X_{2}=\varnothing\end{cases}
\end{aligned}
$$

Since semifuzzy quantifiers are mappings on crisp sets, they are much more intuitive and easier to define than fuzzy quantifiers and are very close to the idea of Zadeh's linguistic quantifier. ${ }^{12}$ Semifuzzy quantifiers lie at a half-way point between crisp quantifiers and fuzzy quantifiers.

A crisp quantifier is formally defined as

DEFINITION 2. ${ }^{6-8}$ An n-ary generalized crisp quantifier on a base set $E$ is a mapping $C Q: \mathcal{P}(E)^{n} \longrightarrow\{0,1\}$.

Examples of crisp quantifiers are

$$
\begin{gathered}
\text { all }\left(X_{1}, X_{2}\right)=X_{1} \subseteq X_{2} \\
80 \% \text { or_more_of_the }\left(X_{1}, X_{2}\right)=\left\{\begin{array}{lr}
\frac{\left|X_{1} \cap X_{2}\right|}{\left|X_{1}\right|} \geq 0.80 X_{1} \neq \varnothing \\
1 & X_{1}=\varnothing
\end{array}\right.
\end{gathered}
$$

Fuzzy quantifiers are formally defined as

DEFINITION 3. ${ }^{6-8}$. An n-ary fuzzy quantifier $\widetilde{F Q}$ on a base set $E \neq \emptyset$ is a mapping $\widetilde{F Q}: \widetilde{\mathcal{P}}(E)^{n} \longrightarrow[0,1]$ which to each choice of $X_{1}, \ldots, X_{n} \in \widetilde{\mathcal{P}}(E)$ assigns a gradual result $\widetilde{F Q}\left(X_{1}, \ldots, X_{n}\right) \in[0,1]$.

${ }^{\text {a}}$ Functions $T_{a, b, c, d}$ and $S_{\alpha, \gamma}$ are defined as usual in the fuzzy literature

$$
T_{a, b, c, d}(x)=\left\{\begin{array}{cc}
0 & x \leq a \\
\frac{x-a}{b-a} & a<x \leq b \\
1 & b<x \leq c \\
1-\frac{x-c}{d-c} & c<x \leq d \\
0 & d<x
\end{array}, \quad S_{\alpha, \gamma}(x)= \begin{cases}0 & x<\alpha \\
2\left(\frac{(x-\alpha)}{(\gamma-\alpha)}\right)^{2} & \alpha<x \leq \frac{\alpha+\gamma}{2} \\
1-2\left(\frac{(x-\gamma)}{(\gamma-\alpha)}\right)^{2} & \frac{\alpha+\gamma}{2}<x \leq \gamma \\
1 & \gamma<x\end{cases}\right.
$$

International Journal of Intelligent Systems $\quad$ DOI 10.1002/int 
An example of a fuzzy quantifier could be $\widetilde{a l l}: \widetilde{\mathcal{P}}(E)^{2} \longrightarrow[0,1]$, which in principle may be defined as

$$
\widetilde{\operatorname{all}}\left(X_{1}, X_{2}\right)=\inf \left\{\max \left(1-\mu_{X_{1}}(e), \mu_{X_{2}}(e)\right): e \in E\right\}
$$

Given expression (2), a certain consensus may be achieved to accept it as a suitable definition for an all expression. Nevertheless, there is usually controversy on the definition of suitable general expressions for outlining the evaluation of a quantified sentence.

The mere definition of semifuzzy quantifiers does not resolve the problem of evaluating fuzzy-quantified sentences. To do so, quantifier fuzzification mechanisms (QFMs) are described in Refs. 6-8 for transforming semifuzzy quantifiers into fuzzy quantifiers, i.e., mappings with domain in the universe of semifuzzy quantifiers and range in the universe of fuzzy quantifiers:

$$
\widetilde{F Q}=F(S F Q)
$$

In Refs. 6-8, a number of QFMs that preserve some relevant axioms and properties derived from them are presented. Also a mechanism based on trivalued cuts on fuzzy sets is defined for obtaining crisp sets in $\mathcal{P}(E)$. This produces some quantification models for which a correct behavior is guaranteed from a theoretical point of view. Also other proposals within this framework were made ${ }^{4,19}$ using other QFM mechanisms and different methods for selecting the crisp sets in $\mathcal{P}(E)$. For some particular cases (type I propositions), these methods collapse into other non-QFM-based approaches. This allows these methods to be seen as a natural extension of previous approaches with strong underlying semantics and a systematic construction method.

In the following sections, applications of fuzzy quantifiers to the fields of information retrieval and mobile robotics navigation are presented. The models that are used for modeling fuzzy quantifiers are the probabilistic method defined in Ref. 19 (which for propositions involving a single fuzzy property collapse into ${ }^{18}$ ) and Zadeh's model, ${ }^{12}$ respectively.

\section{FUZZY QUANTIFICATION IN INFORMATION RETRIEVAL}

Given a document base and an individual who has an information need, the basic IR problem is the quest to find the set of documents, which satisfy the user's information need. IR models differ in the way in which documents and queries are represented and matched. For instance, documents were usually modeled in early systems as bags of terms and Boolean query languages were used for articulating information needs. Nevertheless, there exists much evidence to show that most users cannot articulate effective search statements using Boolean connectives. ${ }^{20}$ This provoked that a number of researchers have explored ways to incorporate some elements of the natural language into the query language. Fuzzy quantification was originally applied for IR in Ref. 13, yielding expressive query languages that 
include linguistic statements implemented through fuzzy quantifiers. Unfortunately, this theoretical proposal was not empirically validated and, hence, its actual impact on retrieval performance is unclear. Experimental results and comparative data are the essence of modern research in IR, and theoretical proposals that were not properly evaluated are open to the criticism that much of the work can be viewed as formalization for its own sake. Indeed, the absence of empirical data has traditionally provoked that the use of fuzzy techniques to assist retrieval systems is not standard in the field. ${ }^{21} \mathrm{~A}$ first step to augment the availability of quantitative empirical data for fuzzy quantification in IR was done in Ref. 22. In this section, we summarize the main experimental findings found in Ref. 22 and we exemplify the advantages that retrieval systems can get from quantified statements.

Consider a query with the form $\operatorname{all}\left(q t_{1}, \ldots, q t_{n}\right)$, where $q t_{i}$ are search terms. In the IR field, the connection between search terms and documents is often weighted using popular heuristics such as $t f / i d f{ }^{23}$ which takes into account the number of appearances of the word in the document ( $t f$, term frequency) and the distribution of the term in the whole document collection (idf, inverse document frequency). Therefore, given a document $d_{k}$ we can straightforwardly obtain a set of $n t f / i d f$ scores, representing the connection between the document's semantics and every search term. A fuzzy quantifier can then be applied for combining the set of $n$ scores provided that the weights are normalized in the interval $[0,1]$.

Formally, given a document $d_{k}$, we define a fuzzy set $C_{d_{k}}$ on the set of query terms applying the popular $t f / i d f$ weighting strategy ${ }^{23}$ :

$$
\begin{aligned}
C_{d_{k}} & =\left\{\mu_{C_{d_{k}}}\left(q t_{1}\right) / q t_{1}, \ldots, \mu_{C_{d_{k}}}\left(q t_{n}\right) / q t_{n}\right\} \\
\mu_{C_{d_{k}}}\left(q t_{i}\right) & =\frac{f_{q t_{i}, k}}{\max _{z} f_{z, k}} \cdot \frac{i d f\left(q t_{i}\right)}{\max _{l} i d f\left(q t_{l}\right)}
\end{aligned}
$$

where $f_{q t_{i}, k}$ is the raw frequency of term $q t_{i}$ in the document $d_{k}$ and $\max _{z} f_{z, k}$ is the maximum raw frequency computed over all terms mentioned by the document $d_{k}$. By $i d f\left(q t_{i}\right)$ we refer to a function computing an inverse document frequency (idf) factor. For instance, it might be defined as $i d f\left(q t_{i}\right)=\log \left(\max _{l} n_{l} / n_{i}\right)$, where $n_{i}$ is the number of documents in which the term $q t_{i}$ appears and the maximum $\max _{l} n_{l}$ is computed over all terms in the indexing vocabulary. The value $i d f\left(q t_{i}\right)$ is divided by $\max _{l} i d f\left(q t_{l}\right)$, which is the maximum value of the function $i d f$ computed over all terms in the alphabet. The idf factor tries to capture how meaningful a term is, taking into account its global frequency in the whole collection. A term that is very frequent in the collection is not a good candidate for discriminating between relevant and irrelevant documents and, thus, it receives a low idf value. On the contrary, very infrequent terms are assigned high idf values because they are potentially good discriminators between relevant and nonrelevant documents. Putting all together, the final $t f / i d f$ value makes that an ideal term is one that appears many times in the involved document $\left(d_{k}\right)$ but very few times in the rest of the document collection.

The fuzzy set $C_{d_{k}}$ models the connection between the document $d_{k}$ and every query component. Quantification can now be applied on $C_{d_{k}}$ for evaluating the 
quantified symbol all. As argued in the previous section, a fuzzy quantifier can be naturally defined from a semifuzzy quantifier through a quantifier fuzzification mechanism. Hence, a key decision is to select the appropriate semifuzzy quantifier which implements every quantification symbol. For instance, a natural way for handling the symbol all is to apply a semifuzzy quantifier which assigns the value 1 if the crisp input set is $\left\{q t_{1}, q t_{2}, \ldots, q t_{n}\right\}$ and 0 otherwise.

If we denote by $Q_{s}$ the semifuzzy quantifier, which supports a given quantification symbol $Q$, the probabilistic fuzzification process is fired on $Q_{s}$ yielding a fuzzy quantifier which is applied on $C_{d_{k}}$. Note that this application in IR involves a single fuzzy set, $C_{d_{k}}$ and the quantification process produces a retrieval score $\left(F\left(Q_{s}\right)\left(C_{d_{k}}\right)\right.$, where $F$ is a QFM.

Different quantifiers can be applied to support different quantification symbols leading to a flexible model in which query term weights can be combined in a variety of ways. Indeed, more evolved combinations of the matching weights can be obtained by varying the definition of the semifuzzy quantifier. This is a strong argument in favor of fuzzy quantification for IR because a wide range of fuzzy quantifiers whose behavior has been deeply studied is available in the literature. This supplies a great variety of methods for combining the query-document weights which goes further away from basic Boolean combinations. For instance, different relaxed mathematical definitions for "at least quantifiers" can be empirically evaluated. Furthermore, it is also interesting to observe that some classical retrieval strategies, such as the inner product matching function of the vector-space model, can also be modeled as particular cases of this general framework. ${ }^{22}$

The selection of particular semifuzzy quantifiers for implementing different quantification symbols is an important decision that affects retrieval performance. For instance, the typical crisp implementation for an at least statement is too rigid to be applied in IR. Given a query with the form at least $4\left(\left\{q t_{1}, q t_{2}, \ldots, q t_{n}\right\}\right)$, it is not fair to consider that a document matching 10 query terms is as good as one matching only four terms. ${ }^{\text {b }}$ Moreover, it is too rigid to consider a document that matches 0 query terms is as bad as one matching three query terms. The intuitions behind at least quantifiers can be good for retrieval purposes if implemented in a relaxed form. In particular, intermediate implementations, between a classical at least and a linear implementation, ${ }^{\mathrm{c}}$ are promising for IR purposes. Indeed, it is not strange that nonrelevant documents match a few query terms simply by chance. To minimize this problem, a relaxed formulation can make that documents matching few terms (less than four for the example depicted above) receive a lower score compared to an alternative linear implementation. On the other hand, unlike the rigid at least implementation, documents matching many terms (more than four for the example) can receive a score that grows linearly with the number of those terms. In general, a number of relaxed formulations may be proposed for every

${ }^{\mathrm{b}}$ Indeed, analogous considerations for Boolean conjunctions and disjunctions provoked that Lee and other researchers were rather critical with the application of fuzzy MIN and MAX operators $^{24,25}$ for IR.

${ }^{\mathrm{c}}$ Note that most popular retrieval matching functions have a linear behavior, that is, they implement a combination of the matching scores that grows linearly with the number of matches. ${ }^{22}$ 
quantification symbol depending on the characteristics of the application domain. For instance, high precision scenarios such as the Web, where the quality of the top-ranked documents is a major issue, would require the application of stricter fuzzy quantifiers. On the other hand, other recall-oriented applications are able to tolerate the presence of some nonrelevant documents at high positions in the rank provided that all relevant material can be found in the rank. ${ }^{d}$ In these cases, softer versions of the fuzzy quantifiers make sense.

\subsection{Information Retrieval Experiments}

The quantifier-based approach for retrieval has been evaluated using standard IR benchmarks. ${ }^{22}$ The experiments were run against a subset of the well-known TREC collection, ${ }^{26}$ which contains more than 175,000 documents and 50 information needs expressed in natural language (called topics). Topics were preprocessed by eliminating common words such as prepositions, conjunctions, etc. (stopword processing) and, afterwards, words are reduced to their syntactical root (stemming). The resulting term stems $t s_{i}$ were used to formulate queries with the form at_least_ $x\left(t s_{1}, t s_{2}, \ldots, t s_{n}\right)$. This sort of query statements is adequate for retrieval purposes because, as argued before, their relaxed implementation incorporates interesting intuitions into the matching process. Since TREC topics are structured into different subfields (title, description, and narrative), different quantifiers can be applied to every subfield and their results can be combined by a boolean connective, e.g., at_least_4(...title terms...) AND at_least_3(...description terms...) AND at_least_3(... narrative terms....). That is, fuzzy quantifiers can be used to tune the relative importance of the individual components of the textual query.

In Ref. 22, the evaluation was centered on the adequacy of different mathematical relaxations for implementing the at least expressions. As argued before, intermediate implementations, between the popular at least and a linear implementation, make sense for retrieval purposes. To validate empirically this claim was the main objective of the evaluation. Figure 1 depicts a typical crisp implementation of an at least statement and the relaxed version applied in the experiments.

For each query, a rank of documents was built and the standard evaluation methodology is applied to get performance metrics. ${ }^{\text {e }}$ The experiments showed clearly that the introduction of quantifiers can lead to improvements in retrieval performance up to $20 \%$ of average precision ${ }^{\mathrm{f}}$ when compared to the inner-product matching function of the vector-space model. ${ }^{g}$ This confirms previous intuitions about the benefits of quantified sentences on retrieval performance. To handle at least expressions through semifuzzy quantifiers with the relaxed form shown

\footnotetext{
${ }^{\mathrm{d}}$ Note that to retrieve every relevant document is a nonrealistic challenge in Web search engines and, hence, recall is not an objective in Web searching.

${ }^{\mathrm{e}}$ Any IR benchmark includes relevance judgments for every topic.

${ }^{\mathrm{f}}$ Precision is an standard performance ratio that helps to measure the goodness of a ranking of documents.

${ }^{\mathrm{g}}$ Recall that this measure can be obtained as a case of the semifuzzy quantifier formalism when a linear semifuzzy quantifier is applied.
} 

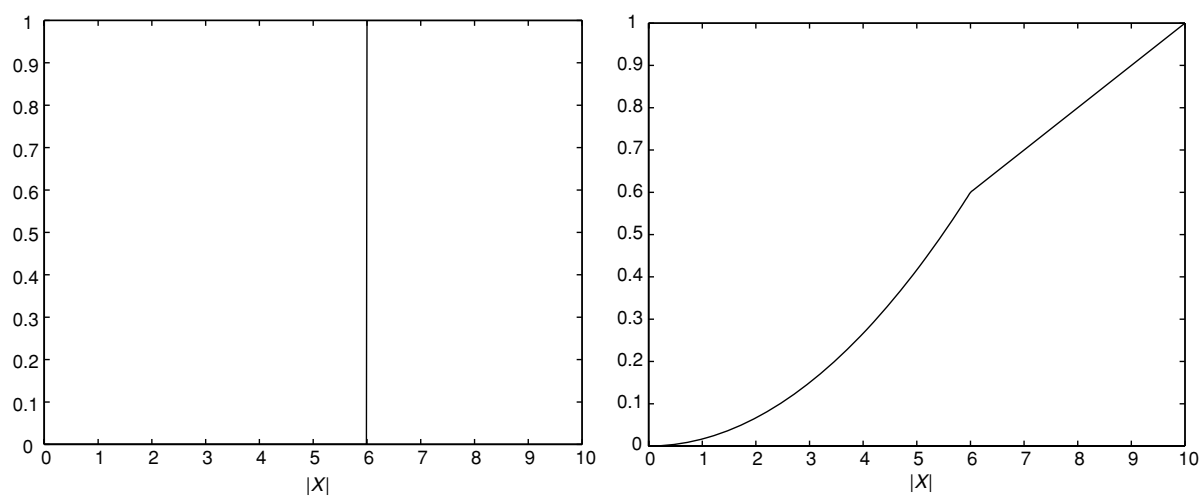

Figure 1. Semifuzzy quantifiers for an at least six expression.

in Figure 1 (right-hand side) outperformed clearly popular retrieval measures. This advances the important role that fuzzy quantification can play in retrieval systems.

Fuzzy quantification appears as a powerful framework in which diverse query quantified statements can be processed leading to a wide range of matching functions. Classical IR approaches tend to oversimplify the content of user information needs, whereas flexible query languages allow to articulate more evolved queries. The simple methods implemented allow to obtain fuzzy representations from TREC topics in an automatic way. This advances that fuzzy query languages might be adequate not only to assist users when formulating their information needs but also to transform textual queries into fuzzy expressions.

\section{FUZZY QUANTIFICATION IN MOBILE ROBOTICS}

The paradigm of fuzzy temporal rules (FTRs) ${ }^{27,28}$ provides a formal model for the representation and reasoning on fuzzy temporal knowledge, oriented to the fields of control/monitoring of systems in real-time applications. The generalization of fuzzy rules to FTRs allows an explicit representation and handling of the occurrence of facts, being this occurrence either absolute or relative to other facts, and also its evolution. Temporal entities such as instants or intervals are considered as well as specification, aggregation, and reduction fuzzy operators.

Within this general framework, a type of simple propositions (fuzzy temporal quantified statements, or FTQS) are used to detect whether certain situations of interest either occur persistently on a given temporal window, in a partially persistent way or at a single temporal point. These propositions involve quantification operators that allow the control action to be taken after considering information on the state of the system within prior temporal instants and not only the current one, as is usual in fuzzy control. FTQS have been used in the field of intelligent control for implementing a fuzzy temporal controller for two behaviors in mobile robotics: wall following ${ }^{29}$ and moving obstacles avoidance. ${ }^{30}$ By means of the analysis of the evolution of state variables throughout temporal references, a 
filtering of noisy sensorial inputs is achieved, and therefore the control action is more reliable. This is a crucial aspect in robotic applications, where uncertainty about measures in the position of static and moving obstacles due to the ultrasound sensors limitations (specular reflection, low angular resolution, and other measurement errors) may be decisive.

\subsection{Fuzzy Temporal Quantified Statements}

Fuzzy temporal quantified statements are syntactically defined as $X$ is $A$ in $Q$ of $T$, where $X$ is a linguistic variable, $A$ represents a linguistic value of $X, T$ is a temporal reference or entity and $Q$ is a fuzzy quantifier.

The temporal entities $T$ may represent both fuzzy temporal instants as well as fuzzy temporal intervals, being in both cases the membership functions defined on a discrete set of values $\tau=\left\{\tau_{0}, \tau_{1}, \ldots, \tau_{k}, \ldots\right\}$, where each $\tau_{k}$ represents a precise temporal instant and $\tau_{0}$ represents the origin. We assume that the values of this set are evenly spaced, where $\triangle=\tau_{j}-\tau_{j-1}$ is the unit of time, whose size or granularity depends on the temporal dynamics of the application that is being dealt with. For robotics $\Delta=\frac{1}{3} s$ has been used, which is the time elapsed between two consecutive control orders.

Different scenarios can occur for the fulfillment of nontemporal part " $X$ is $A$ " when $T$ is an interval: nonpersistence (as in "velocity is high in the last seconds"), where fulfillment is required for at least one point of $T$, persistence (as in "velocity is high throughout the last seconds"), where fulfillment is required throughout the entire interval, and partial persistence, where the nontemporal part should be fulfilled for some subinterval ("in the majority of $T$," "in part of $T$ ").

The execution process of FTQS differs from that of a conventional fuzzy rule in the calculation of the degree of fulfillment (DOF), which now also depends on the prior values of variables. The calculation of DOF is carried in the model in the following manner: in the first place, the degree of fulfillment of the nontemporal part of the proposition is calculated, which is defined as

$$
s c\left(\tau_{k}\right)=\mu_{A}\left(X\left(\tau_{k}\right)\right), \tau_{k} \in \tau
$$

where $\mu_{A}$ is the membership function that is associated with the value $A$ of the proposition, and $X\left(\tau_{k}\right)$ is the value observed for the variable $X$ at the temporal point $\tau_{k}$.

Secondly, sc is modulated by the temporal part of the proposition, so that in all the three cases the weight that is given to the temporal points is proportional to their membership in $T\left(\mu_{T}\right)$ :

- Nonpersistence: " $X$ is $A$ in $T$ "

$$
D O F=\bigvee_{\tau_{k} \in \tau} s c\left(\tau_{k}\right) \wedge \mu_{T}\left(\tau_{k}\right)
$$

- Persistence: " $X$ is $A$ throughout $T$ " 


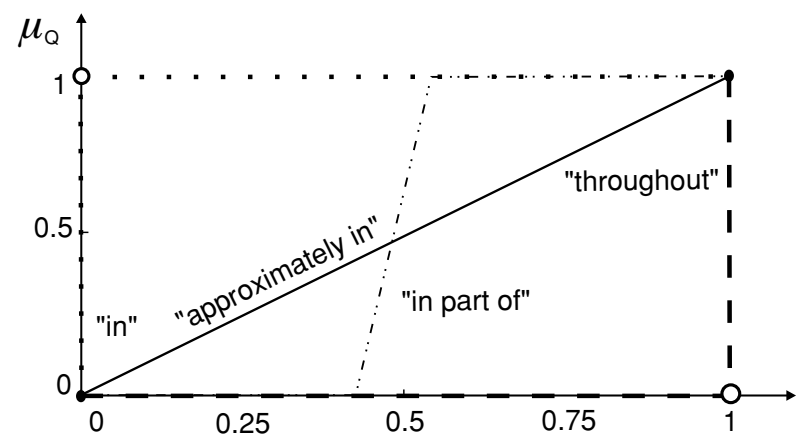

Figure 2. Membership functions $\left(\mu_{Q}\right)$ of some temporal quantifiers.

$$
D O F=\bigwedge_{\tau_{k} \in \tau} s c\left(\tau_{k}\right) \vee\left(1-\mu_{T}\left(\tau_{k}\right)\right)
$$

- Partial persistence: " $X$ is $A$ in $Q$ of $T$ "

$$
D O F=\mu_{Q}\left(\frac{\sum_{\tau_{k} \in \tau} s c\left(\tau_{k}\right) \wedge \mu_{T}\left(\tau_{k}\right)}{\sum_{\tau_{k} \in \tau} \mu_{T}\left(\tau_{k}\right)}\right)
$$

The operators $\wedge$ and $\vee$ are, respectively, the t-norm minimum and the t-conorm maximum, and $\mu_{Q}$ is the membership function that is associated with the linguistic quantifier $Q$. The Quantification model used here is Zadeh's one. ${ }^{12}$

Figure 2 shows the membership functions $\mu_{Q}$ associated with some of the temporal persistence quantifiers used to control the robot ("in," "approximately in," "throughout," and "in part of").

\subsection{Implementation of Behaviors in Mobile Robotics}

For the wall-following behavior, the capacity for including information of a temporal type enables us to filter part of the large amount of sensorial noise and to deal with possible erroneous perceptions of the environment due to unsuitable positioning of the robot. Also, we succeeded in determining the evolution of a variable throughout a temporal interval, due to which it is possible to anticipate future positions of the robot in the environment. In consequence, more continuous behavior patterns are obtained, which enable the maximum velocities to be selected taking into consideration the layout of the environment at each instant, which also results in lower navigation time.

An example of a rule for this behavior is the following one:

IF frontal distance is low in part of the last four measurements AND frontal distance is increasing approximately in the last four measurements AND quotient between the left-hand and frontal distances is high in part of the last four measurements AND velocity is low THEN maintain the velocity 
Table I. Values of some parameters measured during the tests on the real robot.

\begin{tabular}{lccc}
\hline Test & Average Right Distance $(\mathrm{cm})$ & Average Velocity $(\mathrm{cm} / \mathrm{s})$ & Time \\
\hline 1 & 49 & 15 & $3 \mathrm{~m} 35 \mathrm{~s}$ \\
2 & 48 & 11 & $6 \mathrm{~m} 10 \mathrm{~s}$ \\
3 & 45 & 15 & $2 \mathrm{~m} 40 \mathrm{~s}$ \\
4 & 48 & 17 & $2 \mathrm{~m} 53 \mathrm{~s}$ \\
5 & 49 & 16 & $4 \mathrm{~m} 2 \mathrm{~s}$ \\
6 & 47 & 17 & $3 \mathrm{~m} 51 \mathrm{~s}$ \\
7 & 50 & 25 & $4 \mathrm{~m} 1 \mathrm{~s}$ \\
8 & 49 & 25 & $3 \mathrm{~m} \mathrm{55s}$ \\
9 & 52 & 24 & $3 \mathrm{~m} 53 \mathrm{~s}$ \\
10 & 51 & 23 & $4 \mathrm{~m} 7 \mathrm{~s}$ \\
11 & 45 & 18 & $3 \mathrm{~m}$ \\
12 & 48 & 22 & $2 \mathrm{~m} 21 \mathrm{~s}$ \\
13 & 49 & 23 & $\mathrm{~s}$ \\
\hline
\end{tabular}

This rule gets fired in situations where the specular reflection of the ultrasound sensors is very frequent, so measurements are very noisy. For this reason the quantifier "in part of," which requires the fulfillment of the spatial part in at least $50 \%$ of the points in the temporal interval, is used to filter this noise. On the other hand, it is also interesting to know the evolution of the frontal distance. It can be low, but if its values are increasing in the last measurements, the robot could maintain its velocity, although the distance to the wall is low.

Table I shows the values of some parameters (average right distance, average velocity, and time) that have been measured on the real robot in different environments. In all the tests, the average right distance has been very close to the reference distance (the distance at which the robot has to follow the wall is $50 \mathrm{~cm}$ ), although the environments were quite complex (gaps, open doors, corners, etc). This reflects the high reliability and robustness of the control system.

The second of the behaviors that has been implemented is the avoidance of moving obstacles. The majority of approaches proposed in the bibliography only take into account the values of position and velocity of the robot and the obstacle in the present instant. Thus the control action could be not adequate, because the evolution of the moving obstacle in the last instants has not been considered. Imagine two situations in which the moving obstacle is at the same position and with the same velocity, but the first situation has been produced due to an acceleration of the moving obstacle, while the other one has been reached when the obstacle brakes. It is clear that the control action should not be the same for these situations, although at the present instant both moving obstacles have the same state.

For these reasons, the estimation of the trend of the moving object is of special importance to avoid collision. Once the trend has been determined, the controller selects the most adequate behavior for the robot, and implements this behavior with the most suitable values of linear acceleration and angular velocity. FTRs have been used for the estimation of the trend of the moving object. For this estimation, it is necessary to analyze the evolution of the speed and angle of the moving obstacle in the previous instants. One of the major problems for the avoidance is the difficulty to obtain a reliable position and velocity of the moving obstacle (a person, another 


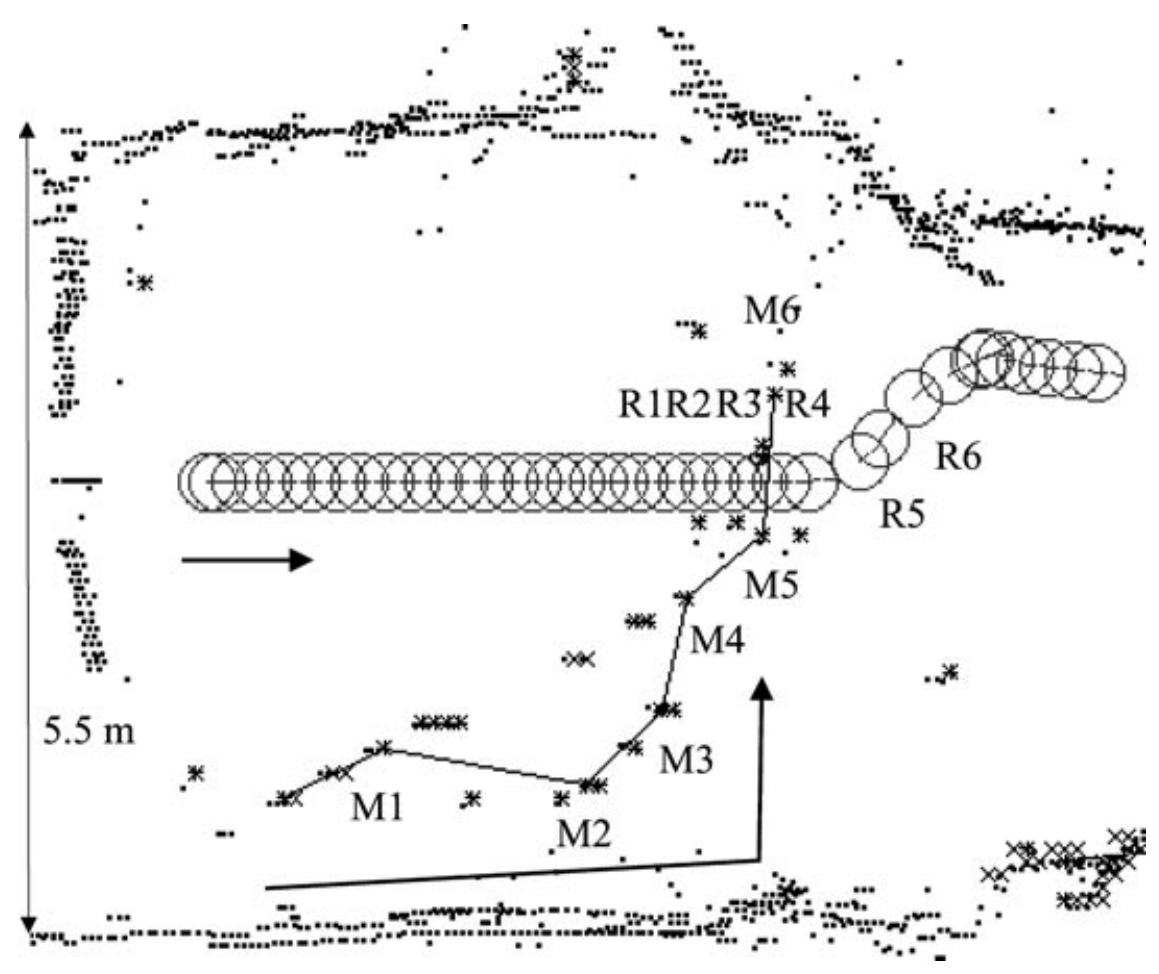

Figure 3. Example of collision avoidance with a person who lets the robot pass.

robot, ... ) due to the low angular resolution of the ultrasound sensors. FTRs help in the filtering of this noise evaluating not only the last position of the obstacle, but the previous ones.

In real environments, and using people as moving obstacles, different tests have been carried out showing that the system is robust and reliable, in spite of the low precision of the method for ultrasonic-based moving obstacle detection. 86 tests have been done (Figure 3 shows one of them) covering a wide range of situations. In $91 \%$ of the situations the robot successfully avoided the collision with the person, and in $79 \%$ of the tests the FTRs correctly estimated the trend of the moving obstacle. The small number of collisions is due to the erroneous estimation of the trend of the obstacle (62\% of the collisions) and the failure in the detection of people with the ultrasound sensors $(38 \%)$.

A real example of collision avoidance is shown in Figure 3. Ultrasonic sensors measurements are represented by points, lines group all detected moving obstacles that correspond to the same existing obstacle, and the robot trajectory is represented by circles.

At the beginning, the robot moves at a constant velocity $(18 \mathrm{~cm} / \mathrm{s})$ and detects the moving obstacle (this happens when the obstacle is placed at M1 and the robot at $\mathrm{R} 1$ ), but takes no action since the trend of the obstacle is indifferent and the collision time is medium, so the robot will observe. Later, when the obstacle is placed at 
M3-M4, the obstacle course evaluation module detects that, due to the obstacle turn to the left, its tendency is to give way. This is done by means of the following rule:

IF collision time is medium AND collision status change is increase in the last three seconds AND nci trend is not decreasing throughout the last two seconds THEN obstacle aim is to give way

This rule analyzes whether variable "collision status change" has taken the value "increase" in at least one point of the temporal reference "the last three seconds," and also that the $n c i$ has not decreased in any point of the temporal reference "the last two seconds." As a consequence, the estimated trend will be "to give way," and the selected behavior will be "to pass in front," which is implemented by means of an acceleration and turning to the left (since obstacle's incidence is from right to left).

\section{CONCLUSION}

In this paper, two real applications of fuzzy quantification in information retrieval and mobile robotics have been presented. This was an attempt to show how fuzzy quantification works under real-world scenarios and the subsequent benefits obtained from a fuzzy quantification based modeling. Indeed, the natural management of linguistic expressions through fuzzy quantifiers is a powerful mechanism able to produce advantages in a wide range of application domains.

The approach to fuzzy quantification applied for information retrieval is based on semifuzzy quantifiers and quantifier fuzzification mechanisms. ${ }^{6-8}$ The evaluation of retrieval performance followed the state of the art methodology of the field of IR and involved a large collection of documents. Experimental results confirmed the high interest of fuzzy quantification techniques for retrieval purposes.

In mobile robotics, we depicted the use of fuzzy quantifiers to evaluate fuzzy temporal quantified expressions. Two behaviors were implemented: contour following and mobile obstacles avoidance, by using a fuzzy temporal control system in which fuzzy quantification plays a fundamental role, since it allows the controller to take into consideration the occurrence of events in previous temporal instants to the current one. This permits the control actions to be more reliable.

\section{Acknowledgments}

Authors acknowledge the support provided by the Spanish Ministry for Science and Innovation under grants TIN2008-00040 and TIN2006-15460-C04-02, by FEDER and Xunta de Galicia (Consellería de Innovación e Industria and Consellería de Educación e Ordeación Universitaria) under grants 07SIN005206PR and 2008/068. Dr. Manuel Mucientes is supported by the Ramón y Cajal program of the Spanish Ministry for Science and Innovation.

\section{References}

1. Barro S, Bugarín A, Cariñena P, Díaz-Hermida F. A framework for fuzzy quantification models analysis. IEEE Trans Fuzzy Syst 2003;11:89-99.

2. Bosc P, Lietard L. Monotonic quantified statements and fuzzy integrals. In: Proc 1994 NAFIPS/IFIS/NASA Conference, 1994. pp 8-12. 
3. Delgado M, Sánchez D, Vila MA. Fuzzy cardinality based evaluation of quantified sentences. Int J Approx Reason 2000;23(1):23-66.

4. Díaz-Hermida F, Bugarín A, Barro S. Definition and classification of semi-fuzzy quantifiers for the evaluation of fuzzy quantified sentences. Int J Approx Reason 2003;34(1):49-88.

5. Díaz-Hermida F, Bugarín A, Cariñena P, Barro S. Voting model based evaluation of fuzzy quantified sentences: a general framework. Fuzzy Sets Syst 2004;146(1):97-120.

6. Glöckner I. DFS — an axiomatic approach to fuzzy quantification. TR97-06, Techn. Fakultät, Universität Bielefeld, 1997.

7. Glöckner I, Knoll A. A formal theory of fuzzy natural language quantification and its role in granular computing. In: W. Pedrycz, editor. Granular computing: An emerging paradigm, volume 70 of Studies in fuzziness and soft computing. Heidelburg, Germany: PhysicaVerlag; 2001. pp 215-256.

8. Glöckner I. Fuzzy quantifiers in natural language: Semantics and computational models. Ph.D. thesis, Universität Bielefeld, 2003.

9. Liu Y, Kerre EE. An overview of fuzzy quantifiers. (i) interpretations. (ii) reasoning and applications. Fuzzy Sets Syst 1998;95:1-121, 135-146.

10. Ralescu AL. Cardinality, quantifiers, the aggregation of fuzzy criteria. Fuzzy Sets Syst 1995;69:355-365.

11. Yager RR. Connectives and quantifiers in fuzzy sets. Fuzzy Sets Syst 1991;40:39-75.

12. Zadeh LA. A computational approach to fuzzy quantifiers in natural languages. Comp. Machs. Appls. 1983;8:149-184.

13. Bordogna G, Pasi G. Linguistic aggregation operators in fuzzy information retrieval. Int $\mathbf{J}$ Intell Syst 1995;10(2):233-248.

14. Herrera-Viedma E, Pasi G. Fuzzy approaches to access information on the web: recent developments and research trends. In: Proc Int Conf on Fuzzy Logic and Technology (EUSFLAT 2003), Zittau, Germany; 2003. pp 25-31.

15. Delgado M, Sánchez D, Vila MA. Acquisition of fuzzy association rules from medical data. In: Fuzzy logic in medicine, volume 83 of Studies in fuzziness and soft computing. Heidelburg, Germany: Physica-Verlag; 2002. pp 286-310.

16. Yager RR. Approximate reasoning as a basis for rule-based expert systems. IEEE Trans Systems, Man Cybern 1984;14(4):636-642.

17. Yager RR. Quantified propositions in a linguistic logic. J. Man-Mach. Stud. 1983;19:195227.

18. Yager RR. On ordered weighted averaging aggregation operators in multicriteria decisionmaking. IEEE Trans Systems, Man Cybern 1988;18(1):183-191.

19. Díaz-Hermida F, Losada DE, Bugarín A, Barro S. A probabilistic quantifier fuzzification mechanism: the model and its evaluation for information retrieval. IEEE Trans Fuzzy Syst 2005;13(5):688-700.

20. Salton G, Fox EA, Wu H. Extended boolean information retrieval. Communi ACM 1983;26(12):1022-1036.

21. Baeza-Yates R, Ribeiro-Neto B. Modern information retrieval. Reading, MA: Addison Wesley, ACM press; 1999.

22. Losada DE, Díaz-Hermida F, Bugarín A, Barro S. Experiments on using fuzzy quantified sentences in adhoc retrieval. In: Proc. SAC-04, the 19th ACM Symposium on Applied Computing-Special Track on Information Access and Retrieval, Nicosia, Cyprus; March 2004. Vol 2, pp 1059-1066.

23. Salton G, McGill MJ. Introduction to modern information retrieval. New York: McGrawHill; 1983.

24. Lee JH, Kim WY, Lee YJ. On the evaluation of boolean operators in the extended boolean framework. In: Proc. of SIGIR-93, the 16th ACM Conference on Research and Development in Information Retrieval, Pittsburgh, PA; 1993.

25. Lee JH. Properties of extended boolean models in information retrieval. In: Proc. of SIGIR94, the 17th ACM Conference on Research and Development in Information Retrieval, Dublin, Ireland: July 1994.

26. TREC: The text retrieval conference. http://trec.nist.gov. Accessed March 30, 2009. 
27. Cariñena P. A model of fuzzy temporal rules for reasoning on dynamic systems. Ph.D. thesis, Universidade de Santiago de Compostela; 2003.

28. Cariñena P, Bugarín A, Mucientes M, Díaz-Hermida F, Barro S. Fuzzy temporal rules: a rule-based approach for fuzzy temporal knowledge representation and reasoning, In: Technologies for constructing intelligent systems. Berlin: Springer-Verlag; 2002. Vol 2, pp 237-250.

29. Mucientes M, Iglesias R, Regueiro CV, Bugarín A, Barro S. A fuzzy temporal rule-based velocity controller for mobile robotics. Fuzzy Sets Syst 2003;134(3):83-99, Special issue on Fuzzy Set Techniques for Intelligent Robotic Systems.

30. Mucientes M, Iglesias R, Regueiro CV, Bugarín A, Cariñena P, Barro S. Fuzzy temporal rules for mobile robot guidance in dynamic environments. IEEE Trans Systems, Man Cybern Part C 2001;33(3):391-398. 